\title{
ANÁLISIS COMPARATIVO ENTRE UNIVERSIDADES ESTATALES Y NO ESTATALES DE CHILE RESPECTO DE SU DIRECCIÓN ESTRATÉGICA
}

COMPARATIVE ANALYSIS BETWEEN STATE AND NON-STATE UNIVERSITIES BELONGING OF CHILEAN REGARDING ITS STRATEGIC DIRECTION

\section{Volumen 15, Número 2}

Mayo - Agosto

pp. $1-20$

Ana María Barra Salazar Nelly Margot Gómez Fuentealba

Revista indizada en REDALYC, $\underline{\text { SCIELO }}$

Revista distribuida en las bases de datos:

CATÁLOGO DE LATINDEX, IRESIE, CLASE, DIALNET, DOAJ, E-REVIST@S, SHERPA/ROMEO, QUALIS, MIAR

Revista registrada en los directorios:

ULRICH'S, REDIE, RINACE, OEI, MAESTROTECA, PREAL, CLACSO 


\title{
ANÁLISIS COMPARATIVO ENTRE UNIVERSIDADES ESTATALES Y NO ESTATALES DE CHILE RESPECTO DE SU DIRECCIÓN ESTRATÉGICA
}

\author{
COMPARATIVE ANALYSIS BETWEEN STATE AND NON-STATE UNIVERSITIES \\ BELONGING OF CHILEAN REGARDING ITS STRATEGIC DIRECTION
}

\author{
Ana María Barra Salazar ${ }^{1}$ \\ Nelly Margot Gómez Fuentealba ${ }^{2}$
}

\begin{abstract}
Resumen: El presente artículo tiene como objetivo analizar los procesos de dirección estratégica de universidades estatales y no estatales chilenas con la finalidad de levantar el estado de situación desarrollado por ellas, establecer comparaciones y evaluar el impacto en sus resultados. Para ello se utilizó el modelo de Wheelen, Hunger y Oliva (2007), el cual consta de cuatro etapas: análisis ambiental, formulación estratégica, implementación estratégica y evaluación y control de la estrategia, esta última, definida en el modelo como evaluación del desempeño. La metodología se adscribe al paradigma de investigación mixta, cualitativa mediante técnicas de investigación documentales provenientes de las páginas Web y de organismos estatales chilenos. Mediante el juicio de expertos se evaluó el grado de avance de las universidades respecto del proceso de planificación estratégica. Los análisis estadísticos incluyen estadística descriptiva, pruebas no paramétricas, análisis de correlación bivariada y modelo de regresión lineal. Los resultados reflejan distintos grados de avance en el proceso de dirección estratégica, detectándose diferencias significativas entre universidades estatales y no estatales en todas las variables del modelo. Asimismo, los datos muestran que a mayor grado de avance en el proceso de planificación estratégica se observa un mayor indicador de calidad de la docencia de pregrado. En adición, el nivel de desarrollo de las universidades estatales respecto de la implementación de la estrategia impacta positivamente en el número de años de acreditación, mientras que para las universidades no estatales el análisis ambiental impacta positivamente en el número de años de acreditación.
\end{abstract}

\section{Palabras clave: PLANIFICACIÓN ESTRATÉGICA, ACREDITACIÓN, EDUCACIÓN SUPERIOR, CHILE}

\begin{abstract}
This article has the objective of analyzing the strategic direction processes of chilean state and nonstate universities in order to gather the state situation developed by them, establish comparison and evaluate the impact in their results. For this, the Wheelen, Hunger and Oliva (2007), model was used, which has 4 stages: environmental analysis, strategic formulation, strategic implementation and evaluation and control of strategy. This last stage is defined in the model as performance evaluation. The methodology is ascribed to the mixed research paradigm, qualitative through techniques of documentary research from websites and chilean state agencies. By expert judgment the progress of universities was evaluated for the strategic planning process. Statistical analysis include descriptive statistics, non parametric tests, analysis of bivariate correlation and linear regression model. Results show different degrees of progress in the strategic direction process, detecting significant differences between state and non state universities in all model variables. The data also show that the higher the degree of progress in the strategic planning process a greater indicator of quality of undergraduate teaching is observed. Moreover, the level of development of state universities regarding the implementation of the strategy positively impacts the number of accreditation years, while for non-state universities environmental analysis positively impacts the number of accreditation years.
\end{abstract}

Keywords: STRATEGIC PLANNING, ACCREDITATION, HIGHER EDUCATION, CHILE

\footnotetext{
1 Docente del departamento de Auditoría y Administración de la Universidad del Bío Bío, Concepción, Chile. Magíster en Ciencias Económicas y Administrativas, Doctora en Administración y Dirección de Empresas. Dirección electrónica: abarra@ubiobio.cl

2 Docente del departamento de Estadística de la Universidad del Bío Bío, Concepción, Chile. Magíster en Estadística. Dirección electrónica: ngomez@ubiobio.cl
}

Artículo recibido: 9 de junio, 2014

Enviado a corrección: 18 de noviembre, 2014

Aprobado: 23 de marzo, 2015 


\section{Introducción}

Las universidades chilenas han presentado cambios profundos durante los últimos veinticinco años. Las transformaciones primordiales han significado un crecimiento notable evidenciado por un aumento de la tasa de cobertura, incremento de la oferta académica, así como la aparición de nuevas universidades privadas, cambios en la estructura de la población estudiantil, aumento en la cantidad de sedes, acceso a nuevas fuentes de financiamiento público, aumento de la competitividad en el mercado en captación de alumnos, atracción y retención de académicos calificados, actualización de planes de estudio, entre otras. Hoy la educación superior representa uno de los sectores de mayor crecimiento en el país (Ministerio de Desarrollo Social, 2006, 2011).

En cuanto a los cambios en las políticas públicas, el Estado ha pasado a ser el principal proveedor de financiamiento de todas las universidades de su propiedad, otorgando recursos por medio de fondos concursables o mediante la asignación de créditos y becas a estudiantes cuya condición socioeconómica así lo amerite. A raíz de estos cambios, las universidades del Consejo de Rectores de las Universidades de Chile (CRUCH), creado el 14 de agosto de 1954 por la ley № 11.575, (Consejo de Rectores Universidades Chilenas, 2013) se vieron enfrentadas a decisiones estratégicas tales como ¿cuánto crecer?, ¿en qué áreas?, ¿en qué regiones?, ¿cuánto invertir?, ¿qué actividades priorizar y cuáles dejar de hacer?, ¿cómo fijar el nivel de los aranceles?, ¿en qué segmento de estudiantes crecer? Asimismo, enfrentaron decisiones con mayor eficiencia y efectividad en las funciones desempeñadas, lo cual fue consecuencia de mercados más competitivos que exigen aumentos de calidad a precios razonables. En este nuevo contexto, aumentan las tensiones en el cumplimiento de los objetivos institucionales, dado que las decisiones de un mayor desarrollo en la misión de la universidad, no necesariamente están asociadas a mayores ingresos para su financiamiento.

Hoy en día, las tendencias de las políticas públicas en la educación superior conducen a tratar de dilucidar las siguientes interrogantes: ¿están cambiando las prácticas de gestión en las universidades chilenas?, ¿en qué medida las universidades del Consejo de Rectores están respondiendo a estos nuevos desafíos de gestión institucional, a través de una profesionalización en su toma de decisiones?, ¿están las universidades utilizando instrumental técnico adecuado que les permita mejorar su gestión en este nuevo entorno más competitivo, cambiante y sujeto a mayor incertidumbre?, ¿se observan cambios en la gestión de recursos humanos, estructura organizacional, funcionamiento de cuerpos 
colegiados, uso de instrumentos técnicos en la evaluación de proyectos y toma de decisiones, entre otros?

Dado este escenario, el rol de la Dirección Estratégica se ha convertido en una fuente de ventaja competitiva de las organizaciones del siglo XXI, ya que uno de sus objetivos más importantes es sobrevivir a la turbulencia y competitividad en la que vivimos, como asimismo a crear valor para la organización y a convertirla en líder en su rubro (Ministerio de Educación de Chile, 2006, 2011).

Por tal razón, hoy en día, en toda empresa surge la necesidad de formular un plan estratégico con el propósito de que la organización mejore sus resultados. Sumado a lo anterior, la Dirección Estratégica de una empresa tiene como finalidad observar y anticipar los desafíos y las oportunidades generadas, tanto de las condiciones externas a la organización, como de su realidad interna. En otras palabras, es un mecanismo de diagnóstico, análisis, reflexión y toma de decisiones colectivas, en torno a la situación actual y futura que deben aspirar las instituciones, esto con el fin de adaptarse a los cambios y a las demandas que les impone el entorno y lograr el máximo de eficiencia y calidad en la prestación de los servicios.

La finalidad de este trabajo es examinar el estado de avance del proceso de Gestión Estratégica y su incidencia en el desempeño en universidades estatales y no estatales vinculadas al Consejo de Rectores de Chile, para finalmente hacer una comparativa respecto de aquellas variables o componentes que son determinantes en los resultados de estas instituciones.

\section{Revisión literaria}

El sistema de dirección ha ido evolucionando, pasando del modelo de la planificación corporativa (años 50-70) al de la planificación estratégica (años 70-80) y de éste a la dirección estratégica (en la actualidad).

La planificación estratégica como sistema gerencial se origina formalmente en los años setenta, como resultado de la evolución del concepto de Planificación, sin embargo, a partir de los años 80 surge el concepto de dirección estratégica con el fin de solucionar diversas necesidades existentes en todas las organizaciones y contribuir a la adaptación de la empresa a las características del entorno. Este concepto es utilizado actualmente en todas las organizaciones (Cabanelas, 1997). 
Para el autor Carnota (1981), la dirección Estratégica es la función de la administración que se ocupa de regular el proceso de cambios que se producen en la empresa, producto de influencias externas e internas, con el propósito de que el sistema organizativo constituido por la propia empresa sea capaz de enfrentar, cada vez con mayor eficiencia, los objetivos.

Teniendo en cuenta el factor humano desde el punto de vista de quien realiza la acción de dirigir y desempeñar la actividad de dirección, los autores Bittel y Ramsey (1997) definen la dirección de empresas como el proceso de integración por medio del cual personas autorizadas crean, mantienen y gestionan una organización en lo que respecta a la selección y cumplimiento de sus objetivos. Los autores Menguzzato y Renau (1991) plantean que los objetivos de la dirección están en la integración y coordinación de esfuerzos de los miembros de la organización, de modo que con ello consigan el logro de las metas trazadas por la entidad y de las personas que la componen.

En este sentido, para Garrido (2006) la Gestión Estratégica como disciplina académica es un planteamiento metodológico de cómo coordinar las acciones de las diferentes partes de una organización con el fin de asegurar su desarrollo y mantenimiento en el espacio y en el tiempo y todo ello en un entorno de competencia. Para Hax y Majluf (1984) la Dirección Estratégica tiene, como objetivo último, el desarrollo de los valores corporativos, las capacidades directivas, las responsabilidades organizativas, y los sistemas administrativos que relacionan la toma de decisiones estratégica y operativa de todos los niveles jerárquicos, y de todas las líneas de autoridad de negocio y funcionales de una empresa.

Sin embargo, todos los autores coinciden en que la Dirección Estratégica es un proceso que no es único para todas las organizaciones, ya que se manifiesta en función de la misión y de las características de cada una de ellas, así como de las pretensiones y las nuevas necesidades que tenga ésta. De igual modo y como lo señalan Navas y Guerra (2007) la planificación estratégica es un instrumento que utilizan los directivos de las organizaciones para dirigirla de manera eficiente, independientemente que sean instituciones públicas o privadas.

Para analizar las universidades y establecer la comparativa entre ellas se utilizó el modelo de Wheelen, Hunger y Oliva, (2007), el cual consta de cuatro etapas: análisis ambiental, formulación estratégica, la implementación estratégica y evaluación y control de la estrategia, esta última definida en el modelo como evaluación del desempeño. Para efectos de este trabajo, se consideró la cuarta etapa como el resultado del proceso que contiene tres 
variables: La calidad en la docencia de pregrado, número de áreas y años de acreditación. A continuación, se describe cada una de las etapas del modelo:

\section{Etapa 1: Análisis Ambiental}

El análisis ambiental se preocupa de la vigilancia, evaluación y difusión de la información de los ambientes internos y externos que afectan a la organización. Su propósito es detectar los factores estratégicos, elementos externos e internos que ayudarán a lograr el éxito de la organización (Wheelen et al., 2007).

\section{Etapa 2: Formulación de la Estrategia}

La formulación estratégica es el segundo paso de la dirección estratégica, es donde se recolecta la mayor parte de la información de las organizaciones, para de esta forma identificar las amenazas y oportunidades del entorno y también las fortalezas y debilidades de la propia compañía. Con este conocimiento las organizaciones están capacitadas para crear sus propias estrategias, aprovechando todos sus recursos, para poder consolidarse como una exitosa organización (Wheelen et al., 2007).

\section{Etapa 3: Implementación de la estrategia}

La implementación estratégica es el cambio donde comienza el verdadero proceso estratégico, constituye una serie de operaciones técnicas que permite poner en marcha la estrategia elegida, siendo muy preciso plasmar las líneas de acción creadas en los planes de acción diseñados, programas y presupuestos; en donde se llevarán a cabo (Wheelen et al., 2007).

\section{Etapa 4: Evaluación y control de la estrategia}

Aquí se analiza detalladamente la formulación e implementación de las estrategias, si están funcionando como se esperaba y los cambios en el ambiente externo. Una vez evaluados estos puntos se puede determinar si la estrategia utilizada puede seguir operando dentro de la organización o si bien, tendrá que ser modificada (Wheelen et al., 2007).

Previamente, se estableció que en este estudio se considera la última etapa como una dimensión de resultado compuesta por las siguientes variables: calidad de la docencia, los años y el número de áreas de acreditación. De este modo, se podrá indicar la incidencia que 
tienen las tres dimensiones anteriores o etapas del modelo en los resultados de las organizaciones en estudio, en este caso, las universidades.

Así el modelo aplicado en el estudio se describe en la Figura 1:

Figura 1

Proceso de Gestión Estratégica y desempeño de Universidades
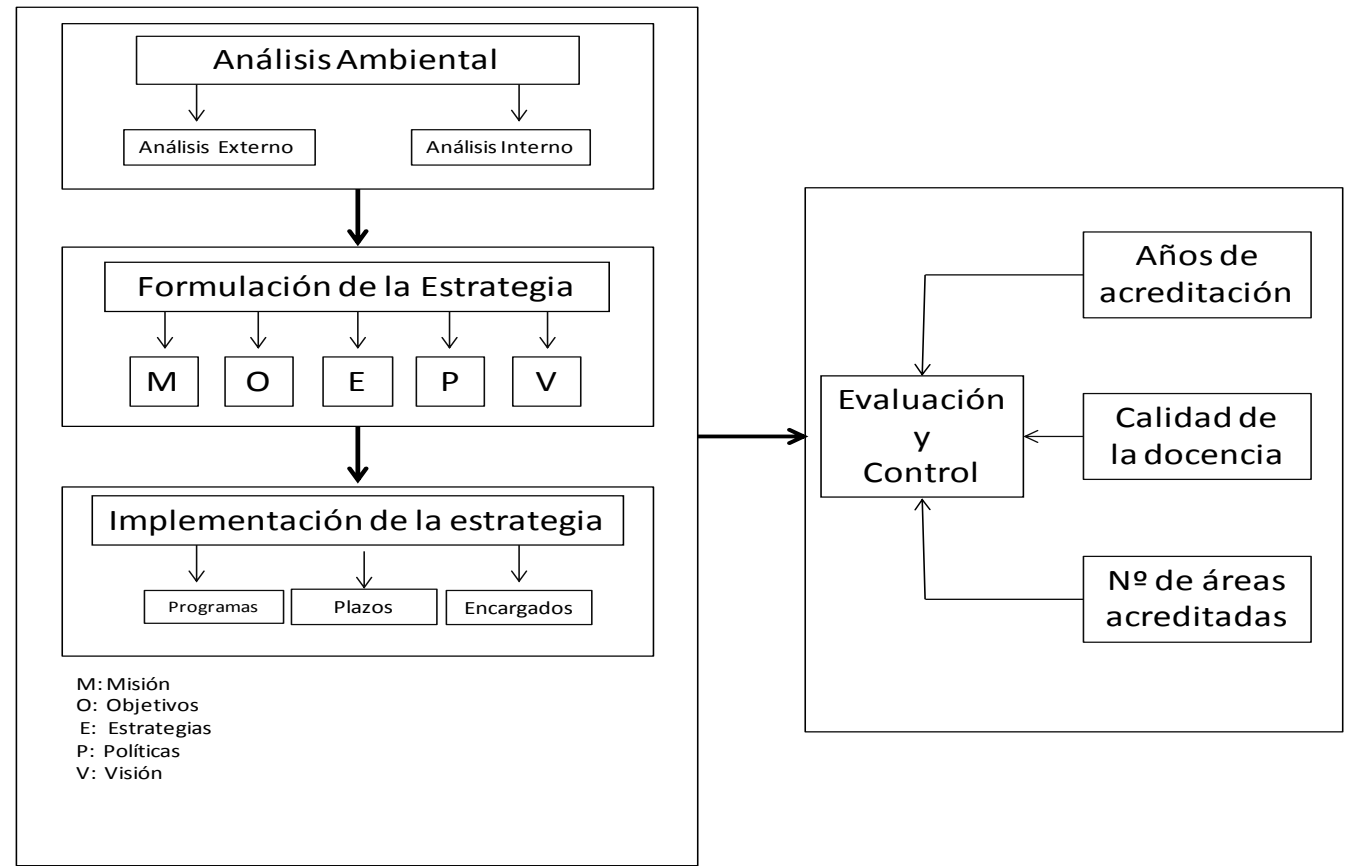

Fuente: Elaboración propia.

\section{Metodología}

\subsection{Descripción de la muestra}

La muestra de estudio comprende 25 universidades, de las cuales el 64\% son universidades estatales y $36 \%$ no estatales, pertenecientes al Consejo de Rectores de las Universidades Chilenas.

Para la elección de las 25 universidades se adoptó el criterio de elegir aquellas con planificaciones estratégicas registradas en su página Web.

A nivel general se puede decir que el estudio abarca diferentes tipos de instituciones que reciben Aportes Fiscales Directos (AFD) de parte del Estado de Chile y cumplen funciones de docencia de pregrado, docencia de postgrado e investigación. No obstante, poseen algunas características que las diferencian de otras, y que tienen que ver con la propiedad, años de acreditación y número de planes estratégicos realizados. El cuadro 1 presenta la distribución de la muestra: 


\section{Cuadro 1}

Distribución de la muestra

\begin{tabular}{|c|c|c|}
\hline Tipo de universidad & No de universidades & Porcentaje \\
\hline Estatal & 16 & $64 \%$ \\
\hline No estatal & 9 & $36 \%$ \\
\hline Total & 25 & - \\
\hline
\end{tabular}

Fuente: Elaboración propia.

\subsection{Instrumentos y Recolección de Información}

Para conocer el proceso de planeación estratégica y el funcionamiento de la gestión institucional se utilizó documentación oficial de las universidades, las cuales están disponibles en sus respectivas páginas Web, donde se encuentra información del plan estratégico y de acreditación. También, se consultaron diferentes organismos gubernamentales dedicados a estudiar y fiscalizar la Educación Superior en Chile, tales como: Ministerio de Educación (MINEDUC), Consejo Nacional de Educación (CNED), División de Educación Superior (DIVESUP), Comisión Nacional de Investigación Científica y Tecnológica de Chile (CONICYT), Departamento de Evaluación, Medición y Registro Educacional DEMRE (DEMRE), Universia, entre otros.

Junto con lo anterior, se utilizó información primaria, como uso de e-mails, visitas y contactos telefónicos con las casas de estudios y en especial, con coordinadores de los procesos de dirección estratégica de cada una de las universidades. El período de referencia para evaluar el proceso de dirección estratégica abarca entre los años 2006 y 2020, tiempo en el cual se encuentran vigentes los distintos planes estratégicos, los cuales oscilan entre 3 y 10 años.

El período de realización de esta investigación fue entre abril y julio de 2012, el cual coincidió con procesos de renovación o actualización de los planes estratégicos en algunas universidades. Respecto del tratamiento estadístico de los datos, éstos se refieren a un análisis descriptivo a través de medidas de centro y variabilidad, análisis comparativo mediante la prueba no paramétrica $U$ de Mann Whitney, análisis de correlación de Spearman y estimación de modelos de regresión múltiple para describir el impacto del proceso de dirección estratégica en el desempeño de las universidades cuantificado por los años de acreditación.

Para los modelos de regresión múltiple se definieron como variables independientes, el "nivel de avance" alcanzado en Análisis Ambiental (AA), Formulación de la Estrategia (FE) e Implementación de la Estrategia (IE).

Se postularon los siguientes modelos para universidades estatales y no estatales: 
Años_Acred est $_{-}=\beta_{0}+\beta_{1} A A_{\text {est }}+\beta_{2} F E_{\text {est }}+\beta_{3} I E_{\text {est }}+\varepsilon$

Años_Acred $d_{\text {noest }}=\beta_{0}+\beta_{1} A A_{\text {nosst }}+\beta_{2} F E_{\text {noest }}+\beta_{3} I E_{\text {nosst }}+\varepsilon$

Donde: $\beta_{0}, \beta_{1}, \beta_{2}$ y $\beta_{3}$ refieren los parámetros desconocidos de la parte determinística del modelo y $\varepsilon$ es el término de error aleatorio que recoge aquellas otras variables que podrían, también, explicar los años de acreditación y que no son consideradas en este modelo.

Para el tratamiento estadístico de los datos se utilizó el software estadístico SPSS versión 19.0 (Statistical Package for the Social Science).

\subsection{Aplicación del instrumento}

Para la obtención de los datos se realizó una evaluación del proceso de planificación estratégica de las universidades a través de una valoración según la escala indicada en el cuadro 2. Para ello, se contó con la opinión de dos expertos quienes evaluaron cada una de las diez variables involucradas en el modelo de la Figura 1. Los valores mínimos y máximos que puede alcanzar cada dimensión en esta escala se resumen en el cuadro 3.

Los datos correspondientes a la dimensión "Evaluación y control" se obtuvieron de información secundaria proveniente de la Comisión Nacional de Acreditación.

\section{Cuadro 2}

Escala de evaluación del grado de avance del plan estratégico universitario

\begin{tabular}{|c|c|c|}
\hline $\mathbf{1}$ & $\mathbf{2 - 3}$ & $\mathbf{4}$ \\
\hline $\begin{array}{c}\text { La variable no se presenta } \\
\text { en el plan estratégico }\end{array}$ & $\begin{array}{c}\text { La variable no se presenta } \\
\text { en su total expresión, o } \\
\text { está formulada con falta de } \\
\text { información. }\end{array}$ & $\begin{array}{c}\text { La variable se presenta en } \\
\text { el plan estratégico y está } \\
\text { bien formulada. }\end{array}$ \\
\hline
\end{tabular}

Fuente: Elaboración propia.

Cuadro 3

Valores mínimos y máximos que puede alcanzar cada dimensión

\begin{tabular}{|l|c|c|c|}
\hline \multicolumn{1}{|c|}{ Dimensión } & $\begin{array}{c}\text { Número } \\
\text { de variables }\end{array}$ & $\begin{array}{c}\text { Puntaje } \\
\text { Mínimo }\end{array}$ & Puntaje Máximo \\
\hline Análisis Ambiental & 2 & 2 & 8 \\
\hline $\begin{array}{l}\text { Formulación de la } \\
\text { Estrategia }\end{array}$ & 5 & 5 & 20 \\
\hline $\begin{array}{l}\text { Implementación de } \\
\text { la Estrategia }\end{array}$ & 3 & 3 & 12 \\
\hline \begin{tabular}{l} 
Total Proceso de Gestión Estratégica \\
\hline
\end{tabular} & 10 & 40 \\
\hline
\end{tabular}

Fuente: Elaboración propia. 
Así, los puntajes mínimos y máximos que pueden presentar las universidades respecto del nivel de avance en sus correspondientes procesos de gestión estratégica fluctúa entre 10 y 40 puntos.

Para medir el porcentaje de concordancia entre los jueces se aplicó el coeficiente de confiabilidad Kappa. Este coeficiente relaciona el acuerdo que exhiben los observadores (o jueces) y corresponde a la proporción de concordancias observadas sobre el total de observaciones habiendo excluido las concordancias atribuibles al azar. El rango de valores que puede tomar se encuentra entre $-1 \mathrm{y}+1$, mientras más cercano $a+1$, mayor es el grado de concordancia entre los jueces. Por el contrario, mientras más cercano a -1 , mayor es el grado de discordancia. Un coeficiente igual a cero indica que la concordancia observada es la que se espera por causa del azar. En este trabajo se generaron tablas de $3 \times 3$ para cada una de las dimensiones y para una evaluación global de las universidades por parte de los jueces. Los resultados se presentan en el cuadro 4.

\section{Cuadro 4}

Valores del coeficiente Kappa por dimensión y global

\begin{tabular}{|c|c|c|}
\hline Dimensión & Kappa por dimensión & Kappa global \\
\hline Análisis Ambiental & 0,76 & \multirow{3}{*}{0,77} \\
\hline Formulación de la Estrategia & 0,71 & \\
\hline $\begin{array}{l}\text { Implementación de la } \\
\text { Estrategia }\end{array}$ & 0,78 & \\
\hline
\end{tabular}

Fuente: Elaboración propia.

Para la interpretación del coeficiente Kappa se utiliza la escala de valoración de Landis y Koch (1977), presentada en el cuadro 5. Esta escala es la que se utiliza con mayor frecuencia para expresar cualitativamente la fuerza de la concordancia entre los observadores. De acuerdo con los valores encontrados en este estudio, se concluye un grado de concordancia sustancial entre ambos jueces.

\section{Cuadro 5}

Escala de valoración del coeficiente Kappa

\begin{tabular}{|c|c|}
\hline Kappa & Grado de acuerdo \\
\hline$<0,00$ & Pobre \\
\hline $0,00-0,20$ & Ligero \\
\hline $0,12-0,40$ & Justo \\
\hline $0,41-0,60$ & Moderado \\
\hline $0,61-0,80$ & Sustancial \\
\hline $0,81-1,00$ & Casi perfecto \\
\hline
\end{tabular}


El comportamiento de las universidades respecto del grado de avance en sus planes estratégicos se analizó mediante distribuciones de frecuencia para cada una de las variables en sus respectivas dimensiones. La comparación de los niveles de avance en el proceso de planificación estratégica entre universidades estatales y no estatales se realizó mediante la aplicación del test no paramétrico $U$ de Mann - Whitney. Esta prueba es una alternativa a la prueba t sobre la diferencia de medias cuando no se cumplen los supuestos de normalidad y homogeneidad de varianzas, o cuando el nivel de medida de los datos es ordinal (Siegel y Castellan, 1995).

Un coeficiente adecuado para medir el grado de asociación entre dos variables que están en escala ordinal es el coeficiente de correlación de Spearman. A través de este indicador se investiga la relación entre cada una de las dimensiones presentes en el proceso de gestión estratégica de la Figura 1. Finalmente, para describir la importancia de estas dimensiones sobre los años de acreditación alcanzado por las universidades se utilizó un modelo de regresión lineal simple.

Si bien, la presente investigación siguió un riguroso método, no se encuentra exenta de limitaciones debido a que el estudio se realizó, principalmente, con datos de fuentes secundarias a partir de los correspondientes sitios Web de las universidades estudiadas, por la misma razón, los modelos de regresión sólo permiten estimar los años de acreditación con valores de las correspondientes variables independientes en el rango de valores de los datos muestrales $\left(3 \leq I E_{\text {est }} \leq 10\right.$ y $\left.5 \leq A A_{\text {no est }} \leq 8\right)$, y no pueden ser vistos como modelos de pronósticos fuera de este rango de valores.

\section{Resultados}

Un análisis general, sin distinguir entre universidades estatales y no estatales, se presenta en el cuadro 6 , del cual se desprende que todas las universidades presentan algún nivel de avance en el análisis interno y externo. Un 56\% de universidades presentan un total grado de cumplimiento en el análisis interno y un $40 \%$ de ellas lo hace para el análisis externo. Respecto de la dimensión Formulación de la Estrategia se observa que sobre un $60 \%$ de universidades muestra un total cumplimiento en la misión y visión, en tanto que entre un $36 \%$ y $48 \%$ lo logra en los objetivos y estrategias. Sin embargo, se observa también que en un $60 \%$ de las universidades, la variable "políticas", no se expresa en su plan estratégico.

Finalmente, en relación con la Implementación de la Estrategia se encuentra que un $52 \%$ de universidades no declaran, explícitamente, los "programas" para la implementación 
de la estrategia, no obstante un $28 \%$ presenta programas bien formulados y acotados para la etapa de ejecución de la estrategia, y sólo un $4 \%$ declara los plazos de cada actividad de su plan estratégico.

\section{Cuadro 6}

Distribución porcentual de las universidades en cada dimensión

\begin{tabular}{|l|l|c|c|c|c|}
\hline \multirow{2}{*}{ Dimensión } & \multicolumn{1}{|c|}{ Variables } & $\mathbf{1}$ & \multicolumn{2}{|c|}{$\mathbf{2 - 3}$} & $\mathbf{4}$ \\
\cline { 2 - 6 } & & $\mathbf{\%}$ & $\mathbf{\%}$ & $\mathbf{\%}$ & $\mathbf{\%}$ \\
\hline Análisis & Análisis interno & 0 & 12 & 32 & 56 \\
\cline { 2 - 6 } ambiental & Análisis externo & 0 & 32 & 28 & 40 \\
\hline \multirow{4}{*}{$\begin{array}{l}\text { Formulación } \\
\text { de la estrategia }\end{array}$} & Misión & 0 & 8 & 24 & 68 \\
\cline { 2 - 6 } & Visión & 0 & 8 & 32 & 60 \\
\cline { 2 - 6 } & Objetivos & 0 & 16 & 36 & 48 \\
\cline { 2 - 6 } & Estrategias & 12 & 20 & 32 & 36 \\
\cline { 2 - 6 } & Políticas & 60 & 28 & 12 & 0 \\
\hline \multirow{3}{*}{$\begin{array}{l}\text { Implementación } \\
\text { de la estrategia }\end{array}$} & Programas & 8 & 12 & 52 & 28 \\
\cline { 2 - 6 } & Procedimientos: Plazos & 20 & 56 & 20 & 4 \\
\cline { 2 - 6 } & Procedimientos: Encargados & 52 & 40 & 8 & 0 \\
\hline
\end{tabular}

Fuente: Elaboración propia.

Respecto de la dimensión "Evaluación y control", el cuadro 7 muestra las medias y desviaciones estándar de las variables: años de acreditación, cantidad de áreas acreditadas y calidad en la docencia de pregrado. Se observa que, en promedio, las universidades se acreditan por 5 años y en 4 áreas incluyendo las dos obligatorias correspondientes a Docencia de Pregrado y Gestión Institucional. En lo que respecta a calidad de la docencia de pregrado, el puntaje promedio se sitúa en 51,88 puntos (en la escala de 1 a 100) con una desviación estándar de 14,24 puntos.

\section{Cuadro 7}

Promedio Evaluación y Control

\begin{tabular}{|c|c|c|}
\hline Evaluación y control & Promedio & $\begin{array}{c}\text { Desviación } \\
\text { estándar }\end{array}$ \\
\hline Años de acreditación & 5 & 1,4 \\
\hline Número de áreas acreditadas & 4 & 1,2 \\
\hline Calidad en la docencia de pregrado & 51,88 & 14,24 \\
\hline
\end{tabular}

Fuente: Elaboración propia.

Se presenta en el cuadro 8 un análisis comparativo entre universidades estatales y no estatales en cada una de las dimensiones consideradas en este estudio y sus correspondientes variables. En cuanto a la variable "Análisis Ambiental" que comprende el análisis del entorno tanto interno como externo se observa que las universidades no estatales han invertido más en acciones publicitarias, logrando con ello un mejor posicionamiento en el mercado. 
Lo mismo ocurre con las variables misión (100\% versus 50\%) y visión (89\% versus 44\%) para universidades no estatales y estatales, respectivamente. Un $56 \%$ de universidades no estatales declara sus objetivos de manera explícita y están bien formulados, mientras que un $31 \%$ de las universidades estatales los declara de una forma más implícita, es decir, que no aparecen en el documento de Planeación.

Por otra parte, se encuentra que un $69 \%$ de universidades estatales no declara expresamente sus políticas en su plan de desarrollo y el $31 \%$ lo hace de forma parcial. Esta cifra baja a un $45 \%$ y $22 \%$ respectivamente, en el grupo de universidades no estatales que cae en esta categoría.

En lo que respecta a la implementación de la estrategia, se observa que un $68 \%$ de universidades estatales versus un $100 \%$ de universidades no estatales expresan programas operativos de implementación y están bien formulados; o bien, están formulados con alguna información faltante. En tanto que un $87 \%$ de universidades estatales y un $56 \%$ de las no estatales o bien no expresan los plazos de implementación en el plan estratégico, o están parcialmente declarados. No se observan universidades (estatales o no estatales) que declaren encargados para la implementación de la estrategia. La mayoría no lo declara en su plan estratégico o lo hace parcialmente, alcanzando a un $94 \%$ de universidades estatales y un $89 \%$ de universidades no estatales en esta categoría.

\section{Cuadro 8}

Distribución porcentual por tipo de universidad

\begin{tabular}{|c|c|c|c|c|c|c|}
\hline \multirow{2}{*}{ Dimensión } & \multirow{2}{*}{ Variable } & \multirow{2}{*}{$\begin{array}{c}\text { Tipo de } \\
\text { Universidad }\end{array}$} & \multirow{2}{*}{$\begin{array}{c}1 \\
\%\end{array}$} & \multicolumn{2}{|c|}{$2-3$} & \multirow{2}{*}{$\begin{array}{l}4 \\
\%\end{array}$} \\
\hline & & & & $\%$ & $\%$ & \\
\hline \multirow{4}{*}{$\begin{array}{l}\text { Análisis } \\
\text { ambiental }\end{array}$} & \multirow{2}{*}{ Análisis interno } & Estatal & 0 & 12 & 44 & 44 \\
\hline & & No estatal & 0 & 11 & 11 & 78 \\
\hline & \multirow{2}{*}{ Análisis externo } & Estatal & 0 & 50 & 19 & 31 \\
\hline & & No estatal & 0 & 0 & 44 & 56 \\
\hline \multirow{10}{*}{$\begin{array}{l}\text { Formulación } \\
\text { de la estrategia }\end{array}$} & \multirow{2}{*}{ Misión } & Estatal & 0 & 12 & 38 & 50 \\
\hline & & No estatal & 0 & 0 & 0 & 100 \\
\hline & \multirow{2}{*}{ Visión } & Estatal & 0 & 12 & 44 & 44 \\
\hline & & No estatal & 0 & 0 & 11 & 89 \\
\hline & \multirow{2}{*}{ Objetivos } & Estatal & 0 & 25 & 31 & 44 \\
\hline & & No estatal & 0 & 0 & 44 & 56 \\
\hline & \multirow{2}{*}{ Estrategias } & Estatal & 19 & 25 & 25 & 31 \\
\hline & & No estatal & 0 & 12 & 44 & 44 \\
\hline & \multirow{2}{*}{ Políticas } & Estatal & 69 & 31 & 0 & 0 \\
\hline & & No estatal & 45 & 22 & 33 & 0 \\
\hline \multirow{6}{*}{$\begin{array}{l}\text { Implementación } \\
\text { de la estrategia }\end{array}$} & \multirow{2}{*}{ Programas } & Estatal & 12 & 19 & 56 & 12 \\
\hline & & No estatal & 0 & 0 & 44 & 56 \\
\hline & \multirow{2}{*}{$\begin{array}{l}\text { Procedimientos: } \\
\text { Plazos }\end{array}$} & Estatal & 25 & 62 & 13 & 0 \\
\hline & & No estatal & 11 & 45 & 33 & 11 \\
\hline & \multirow{2}{*}{$\begin{array}{l}\text { Procedimientos: } \\
\text { Encargados }\end{array}$} & Estatal & 69 & 25 & 6 & 0 \\
\hline & & No estatal & 22 & 67 & 11 & 0 \\
\hline
\end{tabular}

Fuente: Elaboración propia. 
Para establecer comparaciones entre universidades estatales y no estatales, en cada una de las dimensiones, se analiza la variable "nivel de avance", expresado como el puntaje total alcanzado en las tres dimensiones del modelo cuyos valores mínimo y máximo se encuentran en el cuadro 3. En primer lugar, se contrasta la normalidad de los datos mediante el test de Shapiro - Wilk. Este test, publicado en 1965 por Samuel Shapiro y Martin Wilk, se emplea para contrastar la normalidad de un conjunto de datos cuando la muestra es pequeña (menos de 30 datos). Se plantea como hipótesis nula que la muestra proviene de una población normalmente distribuida. Los resultados se presentan en el cuadro 9, donde se muestra el valor del estadístico de Shapiro - Wilk (S-W) tanto para universidades estatales y no estatales y su correspondiente significancia estadística o valor - p. Se observa que la hipótesis de normalidad se rechaza en las universidades estatales para las variables Implementación de la Estrategia y para Análisis Ambiental en las universidades no estatales (valor $-p<0,05$ en ambos casos).

\begin{tabular}{|c|c|c|c|c|}
\hline \multicolumn{5}{|c|}{$\begin{array}{c}\text { Cuadro } 9 \\
\text { Prueba de Normalidad }\end{array}$} \\
\hline \multirow{2}{*}{ Puntaje global } & \multicolumn{4}{|c|}{ Shapiro - Wilk (S-W) } \\
\hline & S-W Estatal & Valor -p & S-W No estatal & Valor - p \\
\hline Análisis ambiental & 0,913 & 0,129 n. s. & 0,767 & $0,009^{* *}$ \\
\hline Formulación de la estrategia & 0,902 & 0,087 n.s. & 0,838 & 0,055 n. s. \\
\hline Implementación de la estrategia & 0,845 & $0,012^{*}$ & 0,896 & 0,231 n. s. \\
\hline
\end{tabular}

De acuerdo con lo anterior, dado que no todas las variables presentan una distribución simétrica unimodal, (distribución de probabilidad normal) y además, debido a que el nivel de medida de los datos es ordinal, se aplica la prueba no paramétrica $U$ de Mann Whitney para la comparación del nivel de avance.

El cuadro 10 muestra los resultados del test, de donde se puede concluir que hay diferencias significativas entre el tipo de universidad respecto de las puntuaciones promedio para cada una de las dimensiones. Esto se traduce en que los niveles de avance en el proceso de planificación estratégica en las dimensiones Análisis Ambiental, Formulación de la Estrategia e Implementación de la Estrategia son diferentes en las Universidades Estatales y no estatales. De manera que a las universidades no estatales les corresponde una manifestación mayor de las variables en sus planes estratégicos pertenecientes a cada dimensión, lo que conduce a concluir que esas universidades presentan un mayor grado de desarrollo en sus planes, como en los procesos de dirección estratégica. 
Cuadro 10

Promedio global y prueba no paramétrica U-Mann-Whitney por tipo de universidad

\begin{tabular}{|c|c|c|c|c|}
\hline Dimensión & Tipo de Universidad & $\begin{array}{l}\text { Media } \pm \\
\text { D.E. }\end{array}$ & $\begin{array}{l}\text { U-Mann } \\
\text { Whitney }\end{array}$ & valor- $\mathbf{p}$ \\
\hline \multirow{2}{*}{ Análisis ambiental } & Estatal & $6,13 \pm 1,36$ & \multirow{2}{*}{38} & \multirow{2}{*}{$0,047^{*}$} \\
\hline & No estatal & $7,22 \pm 1,09$ & & \\
\hline \multirow{2}{*}{ Formulación de la estrategia } & Estatal & $13,88 \pm 3,55$ & \multirow{2}{*}{36,5} & \multirow{2}{*}{$0,043^{*}$} \\
\hline & No estatal & $16,67 \pm 2,17$ & & \\
\hline \multirow{2}{*}{ Implementación de la estrategia } & Estatal & $5,94 \pm 1,65$ & \multirow{2}{*}{25} & \multirow{2}{*}{$0,006^{\star *}$} \\
\hline & No estatal & $7,89 \pm 1,53$ & & \\
\hline
\end{tabular}

*significativo al nivel $0,05{ }^{* *}$ significativo al nivel 0,01

Fuente: Elaboración propia.

Para la dimensión "Evaluación y control", en el cuadro 11 se presentan los resultados de la prueba U-Mann Whitney, la cual compara los puntajes promedio para ambos tipos de universidades, tanto en años de acreditación, cantidad de áreas acreditadas y calidad de la docencia de pregrado. En las tres variables, las universidades no estatales logran una mejor puntuación, observándose diferencias significativas en los años de acreditación (U-Mann Whitney $=29$; valor $-\mathrm{p}=0,013$ ).

\begin{tabular}{|c|c|c|c|c|}
\hline \multicolumn{5}{|c|}{$\begin{array}{l}\text { Cuadro } 11 \\
\text { Promedio Evaluación y Control por tipo de universidad }\end{array}$} \\
\hline \multicolumn{2}{|c|}{ Evaluación y control } & Promedio & $\begin{array}{l}\text { U de Mann } \\
\text { Whitnev }\end{array}$ & valor- p \\
\hline \multirow{2}{*}{ Años de acreditación } & Estatal & 4 & \multirow{2}{*}{29} & \multirow[b]{2}{*}{$0,013^{*}$} \\
\hline & No estatal & 6 & & \\
\hline \multirow{2}{*}{ Áreas de acreditación } & Estatal & 3 & \multirow{2}{*}{43} & \multirow{2}{*}{0,088 n. s. } \\
\hline & No estatal & 4 & & \\
\hline \multirow{2}{*}{$\begin{array}{l}\text { Indicador de calidad } \\
\text { docencia pregrado }\end{array}$} & Estatal & 48,06 & \multirow{2}{*}{43} & \multirow{2}{*}{0,101 n.s. } \\
\hline & No estatal & 58,67 & & \\
\hline
\end{tabular}

${ }^{* *}$ significativo al nivel 0,05 n. s. no significativo

Fuente: Elaboración propia.

Para evaluar la relación entre el nivel de avance del proceso de gestión estratégica y el desempeño de las universidades calculado a través de las variables: años de acreditación, número de áreas acreditadas y calidad de la docencia de pregrado definidas en la dimensión "Evaluación y control", se realiza un análisis de correlación de Spearman entre las variables "nivel de avance" alcanzado en Análisis Ambiental (AA), Formulación de la estrategia (FE), Implementación de la estrategia (IE), Años de acreditación, Número de áreas acreditadas y Calidad de la docencia de pregrado en instituciones estatales y no estatales. Los resultados se presentan, a continuación, en los cuadros 12 y 13 : 
Cuadro 12

Correlación de Spearman para Universidades Estatales

\begin{tabular}{|c|c|c|c|c|c|c|c|}
\hline \multicolumn{2}{|c|}{ Correlación de Spearman } & Calidad & AA & $\mathrm{FE}$ & IE & Años & Áreas \\
\hline \multirow{2}{*}{ Calidad } & Coeficiente de correlación & 1 & $0,51\left(^{*}\right)$ & $0,65\left(^{* *}\right)$ & $0,76\left(^{* \star}\right)$ & $\left.0,82^{* \star}\right)$ & $\left.0,711^{\star \star}\right)$ \\
\hline & Sig. (bilateral) & - & 0,04 & 0,01 & 0,00 & 0,00 & 0,00 \\
\hline \multirow{2}{*}{ AA } & Coeficiente de correlación & & 1 & $0,66\left(^{* *}\right)$ & $0,74\left({ }^{\star \star}\right)$ & $0,55\left(^{*}\right)$ & $\left.0,70^{\star \star}\right)$ \\
\hline & Sig. (bilateral) & & & 0,01 & 0,00 & 0,03 & 0,00 \\
\hline \multirow{2}{*}{ FE } & Coeficiente de correlación & & & 1 & $\left.0,88^{* *}\right)$ & $\left.0,89^{* \star}\right)$ & $\left.0,73^{* \star}\right)$ \\
\hline & Sig. (bilateral) & & & & 0,00 & 0,00 & 0,00 \\
\hline \multirow{2}{*}{ IE } & Coeficiente de correlación & & & & 1 & $\left.0,88^{(*}\right)$ & $\left.0,77^{* \star}\right)$ \\
\hline & Sig. (bilateral) & & & & & 0,00 & 0,00 \\
\hline \multirow{2}{*}{ Años } & Coeficiente de correlación & & & & & 1 & $\left.0,79^{* *}\right)$ \\
\hline & Sig. (bilateral) & & & & & & 0,00 \\
\hline \multirow{2}{*}{ Áreas } & Coeficiente de correlación & & & & & & 1 \\
\hline & Sig. (bilateral) & & & & & & - \\
\hline
\end{tabular}

Fuente: Elaboración propia.

Estos resultados indican, tanto para universidades estatales como no estatales, que la calidad de la docencia de pregrado se correlaciona de manera positiva y significativa con el análisis ambiental, formulación de la estrategia e implementación de la estrategia (en todos los casos el valor- $p<0,05)$. Es decir, los datos muestran que a mayor grado de avance en el proceso de planificación estratégica se observa un mayor indicador de calidad de la docencia de pregrado.

Cuadro 13

\begin{tabular}{|c|c|c|c|c|c|c|c|}
\hline \multicolumn{8}{|c|}{ Correlación de Spearman para Universidades no estatales } \\
\hline \multicolumn{2}{|c|}{ Correlación de Spearman } & Calidad & AA & FE & IE & Años & Áreas \\
\hline \multirow{2}{*}{ Calidad } & Coeficiente de correlación & 1 & $0,76\left(^{*}\right)$ & $0,91\left({ }^{* *}\right)$ & $0,69\left(^{*}\right)$ & $0,94\left({ }^{* *}\right)$ & $0,84\left(^{* *}\right)$ \\
\hline & Sig. (bilateral) & & 0,02 & 0,00 & 0,04 & 0,00 & 0,01 \\
\hline \multirow{2}{*}{ AA } & Coeficiente de correlación & & 1 & $0,79\left(^{\star}\right)$ & 0,36 & $0,81\left(^{\star *}\right)$ & 0,62 \\
\hline & Sig. (bilateral) & & & 0,01 & 0,34 & 0,01 & 0,08 \\
\hline \multirow{2}{*}{ FE } & Coeficiente de correlación & & & 1 & 0,65 & $0,94\left({ }^{* *}\right)$ & $0,92\left(^{* \star}\right)$ \\
\hline & Sig. (bilateral) & & & & 0,06 & 0,00 & 0,00 \\
\hline \multirow{2}{*}{ IE } & Coeficiente de correlación & & & & 1 & $0,70\left(^{*}\right)$ & 0,49 \\
\hline & Sig. (bilateral) & & & & & 0,04 & 0,18 \\
\hline \multirow{2}{*}{ Años } & Coeficiente de correlación & & & & & 1 & $\left.0,81{ }^{* *}\right)$ \\
\hline & Sig. (bilateral) & & & & & & 0,01 \\
\hline \multirow{2}{*}{ Áreas } & Coeficiente de correlación & & & & & & 1 \\
\hline & Sig. (bilateral) & & & & & & - \\
\hline
\end{tabular}

Fuente: Elaboración propia.

Finalmente, en relación con el impacto del proceso de dirección estratégica en el desempeño de las universidades determinado por los años de acreditación, los análisis realizados permiten concluir que en las universidades estatales sólo resulta significativa la variable Implementación de la estrategia, en tanto que para las instituciones de educación superior no estatales es significativa la variable Análisis ambiental. Por lo tanto, los modelos toman la siguiente forma: 
1. Años_Acred est $=0,71 \mathrm{IE}_{\text {est }}$

2. Años_Acred nosst $=0,75$ AA $_{\text {noest }}$

Estos modelos permiten estimar los años de acreditación de universidades estatales y no estatales según el nivel de avance en sus respectivas variables explicativas $\left(I E_{\text {est }} y \quad A A_{\text {no est }}\right)$.

Como se puede observar en el cuadro 14, el programa de implementación de la estrategia desarrollado por las universidades estatales ( $\left.I E_{\text {est }}\right)$ tiene una capacidad explicativa del $82 \%$ sobre los años de acreditación logrados por estas universidades. Asimismo, para el grupo de las universidades no estatales se encuentra que el Análisis Ambiental $\left(A A_{\text {noest }}\right)$ tiene una capacidad explicativa del $87 \%$ sobre los años de acreditación.

Para ambos grupos el Test $F$ confirma la pertinencia estadística de los modelos estimados (valor-p $<0,001)$.

Cuadro 14

Resumen del modelo

\begin{tabular}{|l|l|c|c|c|c|}
\hline $\begin{array}{c}\text { Tipo de } \\
\text { Universidad }\end{array}$ & \multicolumn{1}{|c|}{ Modelo } & $\begin{array}{c}\text { R } \\
\text { cuadrado } \\
\text { corregida }\end{array}$ & $\begin{array}{c}\text { Error típ. } \\
\text { de la } \\
\text { estimación }\end{array}$ & F & valor- p \\
\hline Estatal & 1. variable predictora: IE & 0,82 & 0,57 & 950,63 & 0,00 \\
\hline No Estatal & 2. variable predictora: AA & 0,87 & 0,52 & 1035,39 & 0,00 \\
\hline
\end{tabular}

Fuente: Elaboración propia.

Cuadro 15

Coeficientes del modelo

\begin{tabular}{|l|l|c|c|c|}
\hline \multirow{2}{*}{$\begin{array}{c}\text { Tipo de } \\
\text { Universidad }\end{array}$} & Modelo & $\begin{array}{c}\text { Coeficientes } \\
\text { no } \\
\text { estandarizados }\end{array}$ & \multirow{2}{*}{ T } & \multirow{2}{*}{ valor- $\mathbf{p}$} \\
\cline { 2 - 5 } & 1. variable predictora: IE & B & 30,83 & 0,00 \\
\hline Estatal & 2. variable predictora: AA & 0,71 & 31,18 & 0,00 \\
\hline
\end{tabular}

Fuente: Elaboración propia.

Los resultados en el cuadro 15 permiten concluir que el nivel de desarrollo que presentan las universidades estatales respecto de su programa de implementación de la estrategia impacta positivamente en el número de años de acreditación logrados (Test $\mathrm{t}=$ 30,832; valor-p $<0,001)$.

En cuanto a las universidades no estatales, el desarrollo que presentan respecto de su Análisis interno y externo impacta positivamente en el número de años de acreditación logrados (Test $t=31,177$; valor- $\mathrm{p}<0,001)$. 


\section{Análisis}

En este estudio se han cumplido a cabalidad los objetivos planteados referidos a realizar una comparativa entre los distintos procesos de Dirección Estratégica de las universidades estatales y no estatales, así como se han analizado las variables que componen cada una de las etapas del proceso de Dirección detectándose diferencias entre ellas.

En cuanto a la primera etapa correspondiente a la Dimensión Ambiental, se observa que las universidades expresan en sus planes de desarrollo las variables del medio externo en mayor porcentaje en relación con las variables del ámbito interno.

En la segunda etapa del modelo de Dirección correspondiente a la formulación de estrategias, se observa que las universidades expresan claramente su visión y misión (entre un $60 \%$ y $68 \%$ ), y en menor medida en cuanto a objetivos, estrategias y políticas. En la tercera fase del proceso, pertinente a la Implementación, se observa poco cumplimiento de lo formulado en la etapa dos, lo que significa que las organizaciones, cuentan parcialmente con sistemas que le permitan medir las estrategias que se han puesto en marcha, como programas y procedimientos claros.

En cuanto a las variables que miden el resultado, se observa que en promedio, las universidades se acreditan en cuatro áreas, por un período de 5 años. Al hacer un análisis comparativo entre las instituciones estudiadas, se puede concluir que con respecto al análisis ambiental, las variables de análisis interno y análisis externo se explicitan en mayor grado en las universidades no estatales que en las universidades estatales. En la etapa de formulación de estrategias se observa que, prácticamente, todas las universidades dan cumplimiento de ellas, las estatales se destacan por presentar un mayor grado de desempeño en las variables asociadas a objetivos y estrategias.

En la dimensión "Implementación de la Estrategia", un bajo número de universidades arrojan total cumplimiento en las variables respectivas, lo cual se explica por la dificultad que tiene el seguimiento de la estrategia a través del tiempo en la mayoría de las organizaciones. En términos globales, se observaron diferencias significativas entre las Universidades estatales y no estatales en las tres dimensiones estudiadas.

A través de la prueba $U$ de Mann Whitney para muestras independientes-cuyos resultados se presentan en la Tabla 11-, utilizada para comparar universidades estatales y no estatales tanto en años de acreditación, cantidad de áreas de acreditación y calidad en la docencia de pregrado, se observan diferencias estadísticamente significativas en los años de 
acreditación alcanzados por universidades estatales y no estatales, estas últimas presentan más años y áreas de acreditación, y un mejor indicador de calidad de la docencia de pregrado.

Existe un alto grado de correlación entre las variables estudiadas, la mayor parte de ellas significativas al 1\%. El modelo de regresión lineal múltiple, estimado para las Universidades Estatales, explica un $82,4 \%$ de la variabilidad en los años de acreditación logrados por dichas universidades, manifestado principalmente, por la dimensión Implementación de la Estrategia cuya incidencia es de 0,709.

Respecto de la universidades no estatales, el modelo de regresión lineal múltiple estimado explica un $86,9 \%$ de la variabilidad en los años de acreditación logrados por estas universidades, para el cual la dimensión Análisis Ambiental muestra una incidencia estadística de 0,746.

En general, el puntaje obtenido en Implementación de la Estrategia que poseen las universidades de este estudio tiene un impacto positivo en la calidad de docencia, por lo que se recomienda a las universidades invertir tiempo en desarrollar las actividades que ello implica, como también el seguimiento de las mismas.

\section{Conclusiones generales}

Una vez revisados los antecedentes teóricos, los procesos de dirección estratégica de las universidades estatales y no estatales y, contrastados ambos elementos se puede concluir, según lo establecido por Wheelen et al. (2007), que el modelo de dirección aplicado o formulado por las universidades es distinto en cada una de ellas, como también el grado de cumplimiento de ellos. Las diferencias pueden ser atribuibles tanto a las capacidades y recursos de quienes dirigen la organización como a la escasa experiencia de algunos directivos en problemáticas de alta dirección universitaria (Barra y Gómez, 2014).

Se recomienda a los altos directivos de las universidades trabajar y retroalimentar constantemente el plan estratégico institucional, considerando las variables del entorno y las capacidades y recursos con que cuenta la organización, ya que de esta forma estarán garantizando en gran medida mejores resultados en años de acreditación, calidad de la docencia y número de áreas acreditadas, y por ende una mejor gestión estratégica de su institución. 


\section{Referencias}

Barra, Ana María y Gómez, Nelly Margot. (2014). Relación entre los insumos del plan estratégico y los resultados para la acreditación. Caso aplicado a las universidades chilenas. Revista electrónica Actualidades Investigativas en Educación, 14(3), 1-20. DOI http://dx.doi.org/10.15517/aie.v14i3.16128

Bittel, Lester, Ramsey, Jackson. (1997). Enciclopedia del Management. Barcelona: Grupo Editorial Océano.

Cabanelas, José. (1997). Dirección de Empresas bases en un entorno abierto y dinámico. España: Ediciones Pirámide.

Carnota, Orlando. (1981). Curso de Administración para dirigentes. La Habana: Editorial Ciencias Sociales.

Consejo de Rectores Universidades Chilenas. (2013). Anuario Estadístico. Recuperado de http://www.consejoderectores.cl/web/publicaciones anuario.php

Garrido, Santiago. (2006). Dirección estratégica. Madrid: Editorial McGraw-Hill.

Hax, Arnold, Majluf, Nicolás. (1984). Strategic Management: An Integrative Perspective. Englewood Cliffs: Prentice Hall.

Landis, J. Richard y Koch, Gary G. (1977). The measurement of observer agreement for categorical data. Biometrics, 33(1), 159-174.

Menguzzato, Mirta y Renau, Juan José. (1991). La Dirección Estratégica de la Empresa. Barcelona: Editorial Ariel.

Ministerio de Desarrollo Social, Chile. (2006). Encuesta de Caracterización Socioeconómica Nacional. Casen 2006. Recuperado de http://observatorio.ministeriodesarrollosocial.gob.cl/casen obj.php

Ministerio de Desarrollo Social, Chile. (2011). Encuesta de Caracterización Socioeconómica Nacional. Casen 2011.2 Recuperado de http://observatorio.ministeriodesarrollosocial.gob.cl/casen/casen obj.php

Ministerio de Educación de Chile. (2011). Sitio oficial del Ministerio de Educación. Recuperado de http://www.mineduc.cl

Ministerio de Educación de Chile. (2006). Información general. Recuperado de http://es.wikipedia.org/wiki/Ministerio de Educaci\%C3\%B3n de Chile

Navas, José y Guerra, Luis. (2007). Dirección Estratégica de la Empresa. Barcelona: Editorial Civitas.

Shapiro, S. S., M. B. Wilk. (1965). An Analysis of Variance Test for Normality (Complete Samples). Biometrika, 52,(3/4), 591-611. 
Siegel, Sidney y Castellan, N. John. (1995). Estadística no paramétrica aplicada a las ciencias de la conducta (4a ed.). México: Editorial Trillas.

Wheelen, Thomas, Hunger, David y Oliva, Ismael. (2007). Administración estratégica y política de negocios: conceptos y casos. México: Pearson Education. 